\title{
A Trimming Methodology for a New Coaxial Rotor Configuration of Robotic Helicopter Based on Rotor's Thrust Ratio
}

\author{
Zhi-yan Dong ${ }^{1}$, Shun-an Liu ${ }^{1}$, Yan-li Chen ${ }^{1}$, Jia-lin Liu ${ }^{1}$ and Wen-jian $\mathrm{Wu}^{1}$ \\ ${ }^{1}$ School of Mechanical Science and Engineering, Jilin University, China \\ ${ }^{2}$ Network control laboratory of Electric department, CRRC Changchun Railway \\ Vehicle Co., LTD, China \\ Dongzy13@mails.jlu.edu.cn.lsa@jlu.edu.cn,cylcc@yahoo.com,muyangren757878 \\ 499@163.com,wyfdgg1991@gmail.com
}

\begin{abstract}
A trimming methodology for a robotic new-configuration-coaxial-rotor helicopter was proposed to utilize high efficiency rotor to change the robotic coaxial helicopter movements based on the different rotors' thrust ratio. This configuration cancels mechanical linkages between the upper and lower to decrease the mechanical complexity. It would increase the flexible of control rotor. The integration method was employed to construct a robotic coaxial-rotor helicopter. Blade element momentum theory was adopted to calculate the force and moments of the two rotors. Figure-of-Merits of two rotors were compared to show a high efficiency rotor. This trimming method will contribute to the optimum control allocate strategy of a coaxial rotor helicopter. Consequently, simulation results of frequency response analysis were implemented. It proves that the proposed method can show different effects on state variables. It also can bring an appropriate representation of dynamic response characteristic in open loop. Different effects of upper and lower rotor's cyclic input were discussed. Finally, a control allocation strategy of the coaxial rotor helicopter is suggested.
\end{abstract}

Keywords: Trim method, Modeling, Robotic coaxial-rotor helicopter, Simulation. Control allocation strategy

\section{Introduction}

The coaxial rotor helicopter has re-aroused interest because of its potential to achieve high speed, long range and heavy lift. Many researches have made significant progress on performance analysis, optimum coaxial rotor design and prototype demonstrator [1-3]. Comparing the single rotor helicopter, a coaxial rotor helicopter contains two nearly identical counter-rotating rotors and the tail rotor is eliminated. The general characteristic of a coaxial rotor helicopter is normally complicated mechanical linkage between the upper and lower. However, these complicated mechanical linkage would increase the difficult of rotor design. In addition, these type of configuration will also increase the extra unnecessary mass. Inspired by the idea of eliminating the unnecessary mechanical devices in actuator[4,5], a new configuration coaxial rotor configuration is proposed. The upper and lower rotor is controlled by its own swash-plate dependently in the proposed configuration, respectively. Mechanical linkages between the upper and lower is cancelled. The mechanical constraints of cyclic pitch of the two rotors are released. Each rotor's pitch motion is controlled by its own three mechatronics actuators in Figure 1.

Therefore, it is necessary to manage extra liberated input degrees for this new configuration in trim procedure. As we all know, trim is a vital procedure in helicopter design. It is significantly associated with a control allocate strategy and dynamic response characteristic. A review of the state of the art for the trim formulation has been 
presented [6]. Literature [7] has put forward a traditional four inputs trim method .But there two inputs were fixed before taking-off. This is a consequence of relieving the pilot workload and increasing task performance. On the side, a trim method with six inputs has been implemented to equilibrate the non-linear equation to performance comparison [8]. It was simply assumed that same cyclic pitch inputs of the upper and lower rotor were adopted- Different effects of the cyclic pitch inputs of the upper and lower rotor on the coaxial helicopter movements was neglected.

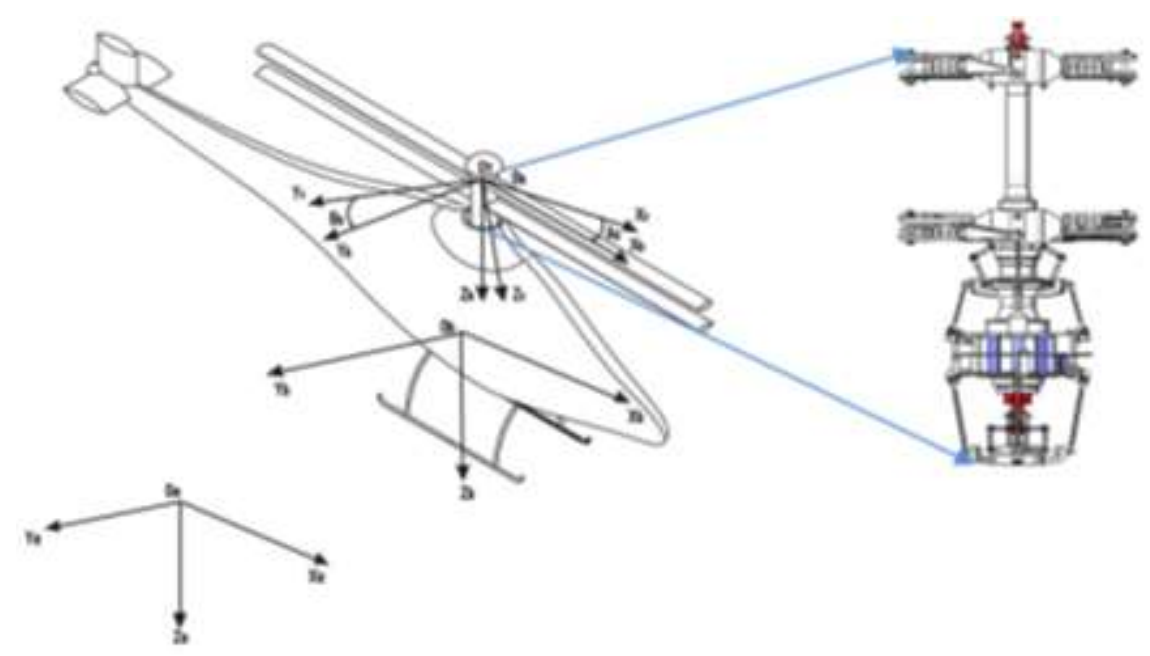

\section{Figure 1. Coaxial Helicopter Prototype with a New Coaxial Rotor Configuration}

However, the previous methods constraint different effects of the cyclic pitch inputs of the upper and lower rotor on the coaxial helicopter movements. The cyclic pitch inputs of the upper and lower rotor aren't seriously considered. From the principle of helicopter, the thrust of rotor is the fundamental origin of movement. To produce a moment is the fundamental principle of cyclic pitch control through tilting the direction of the rotor thrust vector. Therefore, the key ingredient of a new trim methodology design is the extra liberated cyclic pitch inputs of the upper and lower rotors. In the meanwhile, the collective pitches of the upper and lower rotors are varied together to alter the total thrust meeting the requirement of the system. At the same time, zero yawing moment is maintained in our trim methodology. Since the upper and lower rotors flow in different inflow wakes, it brings different thrusts under the same blade airfoil and physical parameter. Furthermore, cyclic inputs are used to control state variables, especially the moment movement. Thus, it is a suggestive idea to utilize these different thrusts to construct a new trim strategy for the designed configuration to consider the different effects on control states. Consequently, thrust ratio of two rotors is introduced to construct relationship of extra liberated upper and lower rotors' cyclic pitch.

Furthermore, simulation model is needed to construct to investigate the dynamic response characteristic of the proposed trim strategy. The state of the art for the formulation of a simulation model has been proposed in [9-11]. Therefore, the integration method was advocated to construct mathematical model of the robotic newconfiguration-coaxial-rotor helicopter.- In predicting thrust and power coefficient of coaxial rotor, it has been validated that blade element momentum theory was almost consistent with free-vortex method prediction [1], which given a better approximation to experimental data using full-scale and model scale coaxial rotors at its operating state and load sharing [12]. Then, BEMT was adopted to generate coaxial rotor's force and moment [13]. 
After constructing the model, it needs a technical tool to evaluate the impact on dynamic characteristics under the proposed trim method. Frequency response analysis has widely been used to validate the dynamic response with flight data in different rotorcraft [14-16]. These applications confirm our confidence that frequency response analysis is indeed superior benefit to dynamic characteristics. Therefore, frequency response analysis was implemented to investigate the dynamic response characteristic of the constructed model. The input degrees totally includes upper and lower rotor's cyclic input, themselves' collective pitch.

The objective of our paper is to propose a trimming methodology for a robotic newconfiguration-coaxial-rotor helicopter to utilize high efficiency rotor based on the rotors' thrust ratio. This methodology includes modeling method, trim strategy, figureof-merit analysis and simulation method. In the modeling method, the constructed coaxial rotor model was regarded as two-main-rotor of a conventional helicopter. The blade element momentum theory was employed to calculate thrusts of the upper and lower rotor under new-configuration-coaxial-rotor. Trim values were work out in analytic method. Stability and control derivatives were derived with assuming that twomain-rotor induced on a rigid body. Moreover, Dynamic characteristic of proposed trim strategy were compared with the existed research. Effects of proposed trimming strategy on trim values were also discussed deeply. Consequently, these endeavors show a confident guide to contribute to a less complicate and lighter coaxial rotor system and more flexible input than the previous work.

\section{Modeling and Trimming}

\subsection{Modeling}

The basic rigid body dynamic and a mechanism for generating force and torque are perfectly assumption for representation of rotorcraft dynamic characteristic and control response $[17,18]$. Consequently, the simulation model was summed up in forces and moments. The model embodies the coaxial rotor, fuselage, vertical empennage and horizontal stabilizer. The forces and moments act along the bodyfixed axes system that centered at the helicopter's center of mass. Figure 1 illustrates the body-fixed axes system with $\mathrm{x}$-axis forward, $\mathrm{y}$-axis rightward and $\mathrm{z}$ axis to the down. The $\mathrm{O}_{e}, \mathrm{O}_{b}, \mathrm{O}_{t}$ and $\mathrm{O}_{h}$ designate the origin of earth coordinate, body coordinate, tip-path plane coordinate, hub plane coordinate, respectively. Furthermore, a rigid body dynamics is generally described by the Newton-Euler equations of motion-as followed:

$$
\begin{gathered}
{\left[\begin{array}{l}
\dot{u} \\
\dot{v} \\
\dot{w}
\end{array}\right]=\left[\begin{array}{l}
-g \sin \theta \\
g \sin \phi \cos \theta \\
g \cos \phi \sin \theta
\end{array}\right]+\frac{1}{m}\left[\begin{array}{l}
X \\
Y \\
Z
\end{array}\right]-\omega^{*}\left[\begin{array}{l}
u \\
v \\
w
\end{array}\right]} \\
{\left[\begin{array}{l}
\dot{p} \\
\dot{q} \\
\dot{r}
\end{array}\right]=-I^{-1} \omega^{*} I\left[\begin{array}{l}
p \\
q \\
r
\end{array}\right]+I^{-1}\left[\begin{array}{c}
R \\
M \\
N
\end{array}\right]} \\
\text { There, } \omega^{*}=\left[\begin{array}{ccc}
0 & -r & q \\
r & 0 & -p \\
-q & p & 0
\end{array}\right], I=\left[\begin{array}{ccc}
I_{x x} & 0 & -I_{x z} \\
0 & I_{y y} & 0 \\
-I_{x z} & 0 & I_{z z}
\end{array}\right],(X, Y, Z),(R, M, N) \text { is }
\end{gathered}
$$

resultant force and torque respectively calculated in the following way: 


$$
\begin{aligned}
& \left(\begin{array}{l}
X=X_{u p}+X_{l o w}+X_{H}+X_{V}+X_{F} \\
Y=Y_{u p}+Y_{l o w}+Y_{V}+Y_{F} \\
Z=Z_{u p}+Z_{\text {low }}+Z_{H}+Z_{V}+Z_{F}
\end{array}\right) \\
& \left(\begin{array}{l}
R=R_{u p}+Y_{u p} h_{u p}+Z_{\text {up }} y_{u p}+R_{\text {low }}+Y_{\text {low }} h_{\text {low }}+Z_{\text {low }} y_{\text {low }}+Y_{V} h_{V}+Y_{F} h_{F}+R_{F} \\
M=M_{u p}-X_{\text {up }} h_{\text {up }}+Z_{\text {up }} l_{\text {up }}+M_{\text {low }}-X_{\text {low }} h_{\text {low }}+Z_{\text {low }} l_{\text {low }}-X_{H} h_{H}+Z_{H} l_{H}-X_{V} h_{V}+M_{F}+Z_{F} l_{F}-X_{F} h_{F} \\
N=N_{u p}-Y_{\text {up }} l_{\text {up }}-X_{\text {up }} \text { up }_{\text {up }}-N_{\text {low }}-Y_{\text {low low }}-X_{\text {low }} y_{\text {low }}-Y_{V} l_{V}+N_{F}-Y_{F} l_{F}
\end{array}\right)
\end{aligned}
$$

Where subscripts up, low, $H, V$ and $F$ indicates upper rotor, lower rotor, horizontal empennage, vertical fin and fuselage, respectively. $l, y, h$ stand for distance that each component respects to center of the body-fixed axes in $\mathrm{x}$-axis, $\mathrm{y}$-axis, $\mathrm{z}$-axis.

\subsection{The Coaxial Rotor}

The coaxial rotor is the key ingredient to construct a simulation model. The forces and moments of the two rotors are calculated dependently with accounting for the interference effects by linear superposition which the upper rotor's slipstream wake interferes the inflow of lower, but the lower rotor doesn't affect the upper rotor's inflow[1]. Thus, blade element theory with the assumptions of two-dimensional and steady airfoils was applied to model the coaxial rotor. Hence, the thrust and torque was calculated as followed:

$$
\left(\begin{array}{l}
T_{u p}=\rho A(\Omega R)^{2} C_{T_{u p}} \\
T_{l o w}=\rho A(\Omega R)^{2} C_{T_{l o w}} \\
Q_{u p}=\rho A(\Omega R)^{2} R C_{T_{u p}} \\
Q_{l o w}=\rho A(\Omega R)^{2} R C_{T_{u p}}
\end{array}\right)
$$

Where $T$ and $Q$ stand for thrust and torque, air density is representation of $\rho$, disc area is written in terms of $A$, rotational speed of rotors is indicated by $\Omega, R$ is radius of the rotor. $C_{T}$ and $C_{Q}$ denote thrust coefficient and torque coefficient. Based on blade element theory, the thrust and torque coefficient of the coaxial rotor are obviously conceived around the rotor azimuth and over the blade span and numerically integrated across each respective rotor disk that blade was discretized into a series of small radial constant elements. The upper rotor whose advancing blade is on the right side, and the lower rotor is clock-wise.

In the first instance, calculating of the upper rotor' thrust and torque is considered. The inflow velocity and incremental thrust with adaptions is derived as[13].

$$
\lambda_{u p}=\sqrt{\left(\frac{\sigma_{u p} C_{l}}{16 F}\right)^{2}+\frac{\sigma_{u p} C_{l}}{8 F} \theta_{u p} r}-\left(\frac{\sigma_{u p} C_{l}}{16 F}\right)
$$

Thrust coefficient $C_{T}$ and power coefficient $C_{P}$ [19] are defined as

$$
\begin{array}{r}
d\left(C_{T}\right)_{u p}=\frac{\sigma_{u p} C_{l}}{2}\left(\theta_{u p} r^{2}-\lambda_{\text {up }} r\right) d r \\
\left(C_{P}\right)_{u p}=\int_{r=0}^{r=1} \lambda_{u p} d\left(C_{T}\right)_{u p}+\int_{0}^{1} \frac{1}{2} \sigma_{u p} C_{d} r^{3} d r
\end{array}
$$

To develop present analysis further, the thrust and torque's coefficient of upper rotor was numerically integrated across each respective rotor disk. Blade was discretized into a series of small radial constant elements. Torque's and power's coefficients are numerically equal. 
To calculate thrust and torque of the lower rotor, the mathematical principles same as the upper rotor-was adopted. However, lower rotor is combined reasonably with two approximation parts, one for the inner part encountering by fully slipstream wake of the upper rotor, and the other outer part freely affecting by the slipstream wake. The boundary of two parts was defined on the basis of wake's assumed radial contraction of the upper rotor ideally or empirically. To this end, the inflow velocity and incremental thrust of lower rotor is followed:

For $r \leq a$

$$
\lambda_{\text {low }}=\sqrt{\left(\frac{\sigma_{l o w} C_{l}}{16 F}-\frac{\lambda_{u p}}{2 a^{2}}\right)^{2}+\frac{\sigma_{l o w} C_{l}}{8 F} \theta_{l o w} r}-\left(\frac{\sigma_{u p} C_{l}}{16 F}-\frac{\lambda_{u p}}{2 a^{2}}\right)
$$

$\mathrm{r}>\mathrm{a}$

$$
\begin{gathered}
\lambda_{\text {low }}=\sqrt{\left(\frac{\sigma_{\text {low }} C_{l}}{16 F}\right)^{2}+\frac{\sigma_{l o w} C_{l}}{8 F} \theta_{l o w} r}-\left(\frac{\sigma_{l o w} C_{l}}{16 F}\right) \\
d\left(C_{T}\right)_{l o w}=\frac{\sigma_{l o w} C_{l}}{2}\left(\theta_{l o w} r^{2}-\lambda_{\text {low }} r\right) d r
\end{gathered}
$$

Note that $a$ is factor which maximum radial station of upper rotor wake imping on lower rotor. Thrust coefficient $C_{T_{\text {low }}}$ and power coefficient $C_{P_{\text {low }}}$ are defined as

$$
\begin{gathered}
\left(C_{T}\right)_{\text {low }}=\int_{r=0}^{r=1} d\left(C_{T}\right)_{\text {low }} \\
\left(C_{P}\right)_{\text {low }}=\int_{r=0}^{r=1} \lambda_{\text {low }} d\left(C_{T}\right)_{\text {low }}+\int_{0}^{1} \frac{1}{2} \sigma_{\text {low }} C_{d} r^{3} d r
\end{gathered}
$$

Based on blade element theory, it is recognized that thrust and torque of the upper and lower rotor are obviously conceived around the rotor azimuth and over the blade span. The generation method of coaxial rotor thrust and torque laid a foundation for trimming procedure.

In the meanwhile, modeling conventional helicopter's fuselage-was expanded to model fuselage of a robotic coaxial rotor helicopter in terms of sideslip and incidence. The vertical fin and horizontal empennage also adopted the modeling method in the single rotor helicopter [20, 21]. It was supposed that the force and moment of the fuselage only downwash affected by lower rotor, but upper rotor doesn't. Scaling the force and moment coefficient of the existed fuselage was applied to calculation of force and moment using the dimensions of constructed model. This physical parameter can be automatic calculated by the prototype of CAD schematic.

The moments and forces of the vertical fin and horizontal empennage play important roles in yawing moment equilibrium equation and longitudinal trim conditions, respectively. However, this effect is evident in the real flight, but not well predicted by simulation[22]. Thus, the effect of the vertical fin, horizontal empennage force and moment was neglected. It is possible to employ the same thought extending to modeling of the vertical fin and horizontal empennage as fuselage in the constructed model[23].

Furthermore, the fidelity level of constructed model, from the view of buildup in Ref.[22], belongs to the between level 1 and level 2. It has an ability to represent underlying physics and to interpret cause and effect of model behavior. Therefore, these gain our confidence to carry on following work.

\subsection{Trimming}

Trimming is situation where forces and moments are in equilibrium about the center of gravity in operating steady flight. Trim strategy concerns suitable input control positions. It is required to solve the non-linear equilibrium equation in the specified flight condition. 
Therefore, the collective pitchs of the upper and lower rotors are varied together to alter the total thrust meeting the requirement of the system. At the same time, zero yawing moment was maintained on the non-linear model in our trim strategy. Our design aim mainly focused on the requirement for weight of the helicopter with $200 \mathrm{~kg}$. Other parameters was referred in [10]. Moreover, the upper and lower rotor adopted the teetering two-blade configuration. Blades are identical in blade sections employing NACA0012, radius, plan-form, twisted rates and cut-out in Table 1. Consequently, the thrust of the upper and lower rotors is $1141.5(\mathrm{~N})$ and $881(\mathrm{~N})$.

\section{Table 1. Physical Parameters of the Coaxial Helicopter}

\begin{tabular}{c|c|c|c|c|c}
\hline$R$ & $e$ & $c$ & $T_{r}$ & $T_{w}$ & $h$ \\
\hline $1.8 \mathrm{~m}$ & $0.1 \mathrm{~m}$ & $0.15 \mathrm{~m}$ & $1: 1$ & $-6^{\circ}$ & $0.2 \mathrm{~m}$ \\
\hline
\end{tabular}

In order to establish the square equilibrium equation, trimming strategy is proposed based on the rotor's thrust ratio for the proposed configuration coaxial rotor helicopter. That is to say, the cyclic inputs between the upper and lower rotor was defined as proportional to the rotor's thrust as followed:

$$
\left(\begin{array}{l}
\frac{\left(a_{1 s}\right)_{u p}}{\left(a_{1 s}\right)_{l o w}} \propto \frac{T_{u p}}{T_{l o w}} \\
\frac{\left(b_{1 s}\right)_{u p}}{\left(b_{1 s}\right)_{l o w}} \propto \frac{T_{u p}}{T_{l o w}}
\end{array}\right)
$$

Where, $a_{1 s}$ is first lateral harmonic coefficient of blade flapping. First longitudinal harmonic coefficient of blade flapping is indicated by $b_{1 s}$. Furthermore, substituting equation (14) into equation (3),(4) and then into equation (1),(2). Therefore, the square equilibrium equations are obtained. In addition, the spin mode that its' axis always directing vertically is driven into generally prescribing operating flight condition. Finally the solution of the trim condition were implemented analytically. The trim values are respectively made lists in Table 2 . The advantage of the proposed trim method is that high figure of merit (FOM) of the upper and lower rotor is utilized. The single rotor FOM is defined as[24]:

$$
\mathrm{FOM}=\frac{C_{T}^{3 / 2}}{\sqrt{2} C_{Q}}
$$

Consequently, FOM of the upper and lower rotor is 0.7316 and 0.4961 , respectively. It indicates that the upper rotor is more efficient than lower rotor. This is because the extra lower rotor' induced power is lost to compensate for the large downwash wake interference from the upper rotor. In terms of collective pitch control, the lower rotor collective input is also little higher than the upper rotor. The higher collective pitch of the lower rotor is in better harmony with the findings from other trimmed coaxial rotor in hover condition[12, 25]. In addition, thrust sharing ratio of the both rotors shows agree well with the previous work. Meanwhile, different FOMs of the two rotors show that the proposed trim method is effective in distinguishly treating cyclic input of the upper rotor. 
Table 2. Trim Inputs and State Variables

\begin{tabular}{c|c}
\hline Inputs/states & Parameter \\
\hline$\theta$ & 0.1147 \\
$\phi$ & -0.1249 \\
$\left(B_{1}\right)_{u p}$ & -0.1285 \\
$\left(A_{1}\right)_{u p}$ & 0.1367 \\
$\left(B_{1}\right)_{\text {low }}$ & 0.0991 \\
$\left(A_{1}\right)_{\text {low }}$ & 0.1055 \\
$\left(\theta_{\text {col }}\right)_{\text {up }}$ & 0.242 \\
$\left(\theta_{\text {col }}\right)_{\text {low }}$ & 0.2731 \\
\hline
\end{tabular}

\section{Dynamic Stability}

\subsection{Linearize}

After trimming non-linear model, extracting linear model is implemented at operating condition. The change of dynamic system in trim condition can be depicted by superposition of individual effects of changes of the independent variables. Moreover, kinematic equations are applied to described the relative rotation motions between the body-fixed frame and the earth frame in the following way:

$$
\left[\begin{array}{l}
\dot{\theta} \\
\dot{\phi} \\
\dot{\psi}
\end{array}\right]=\left[\begin{array}{ccc}
0 & \cos \phi & -\sin \phi \\
1 & \sin \phi \tan \theta & \cos \phi \tan \theta \\
0 & \sin \phi \sec \theta & \cos \phi \sec \theta
\end{array}\right]\left[\begin{array}{l}
p \\
q \\
r
\end{array}\right]
$$

It is impressive hypothesis that linear effects are respectively added up The input variable effects are also considered. Finally, small perturbation theory is embraced, which is rigorously true for small independent variables from the trim values. It is more importantly valid enough to use for the stability analysis. Linear model is derived as follow:

$$
\dot{\mathbf{x}}=\mathbf{A} \mathbf{x}+\mathbf{B u}
$$

Here, state and control matrices are corresponding written in terms of $\mathrm{A}$ and $\mathrm{B}$. Stability and control derivatives are evaluated by taking use of the numerical linearization form. The significant rotors derivatives are showed in Table 3 .

Table 3. Basic Rotors' Derivatives

\begin{tabular}{ccc}
\hline Deriavtives & Expressions & values \\
\hline$\frac{\partial a_{1 s}}{\partial \mu}$ & $\frac{8}{3} \theta_{0}+2 \theta_{1}-2 \frac{v_{1}}{\Omega R}$ & upper: 0.342 \\
$\frac{\partial b_{1 s}}{\partial \mu}$ & $a_{0 u p}=\frac{2}{3} \gamma \frac{C_{T} / \sigma}{\mathrm{a}} \frac{(1-e / R)^{2}}{1+e / R}$ & lower:0.437 \\
$\frac{\partial C_{T} / \sigma}{\partial \lambda^{\prime}}$ & $1 / \frac{8}{a}+\frac{\sqrt{\sigma / 2}}{\sqrt{C_{T} / \sigma}}$ & Upper:0.0047 \\
\hline
\end{tabular}




\begin{tabular}{|c|c|c|}
\hline$\frac{\partial C_{Q} / \sigma}{\partial \lambda^{\prime}}$ & $-\frac{a}{4}\left(\theta_{0.75}-2 \frac{v_{1}}{\Omega R}\right)$ & $\begin{array}{l}\text { Upper: }-0.01 \\
\text { Lower: }-0.074\end{array}$ \\
\hline$\partial a_{1 s} \partial b_{1 s}$ & $16--12 e / R$ & \\
\hline$\overline{\partial q} \overline{\partial p}$ & $\overline{\Omega(1-e / R)^{2}} \overline{\gamma \Omega(1-e / R)^{3}}$ & -0.054 \\
\hline$\frac{\partial a_{1 s}}{\partial p}-\frac{\partial b_{1 s}}{\partial q}$ & $\frac{1}{\Omega}\left[1-\frac{192 e / R}{\gamma^{2}(1-e / R)^{5}}\right.$ & 0.0027 \\
\hline$\frac{\partial a_{1 s}}{\partial A_{1}} \frac{\partial b_{1 s}}{\partial B_{1}}$ & $\frac{12 e / R}{\gamma(1-e / R)^{3}}$ & 0.19 \\
\hline$\frac{\partial a_{1 s}}{\partial B_{1}}-\frac{\partial b_{1 s}}{\partial A_{1}}$ & $-1 / 1+\frac{144(e / R)^{2}}{\gamma^{2}(1-e / R)^{6}}$ & -0.965 \\
\hline$\underline{\partial C_{H} / \sigma} \partial C_{y} / \sigma$ & $\underline{3} C_{\tau} / \sigma\left(1-\underline{\mathrm{a}} \underline{\theta_{0.75}}\right)$ & Upper : 0.07985 \\
\hline$\partial a_{1 s} \quad \partial b_{1 s}$ & $\overline{2}_{T} / \sigma\left(1-\overline{18} \overline{C_{T} / \sigma}\right)$ & Lower:0.0331 \\
\hline$\frac{\partial M}{\partial} \frac{\partial R}{\Omega}$ & $\underline{3} \underline{e} \underline{A_{b} \rho R(\Omega R)^{2} \mathrm{a}}$ & 1719.2 \\
\hline $\begin{array}{l}\partial a_{1 s} \partial b_{1 s} \\
\frac{\partial \mu}{\partial \dot{x}}\left(\frac{\partial \lambda^{\prime}}{\partial \dot{z}}\right)\end{array}$ & $\begin{array}{lc}\overline{4} \bar{R} & \gamma \\
\left(\frac{1}{\Omega R}\right) & 83.7 \mathrm{rad} / \mathrm{s}\end{array}$ & $1 / 150.7=0.00663$ \\
\hline
\end{tabular}

The 8-dimensional state vector is

and the input variables is

$$
\mathbf{x}=[u v w p q r \theta \phi]
$$

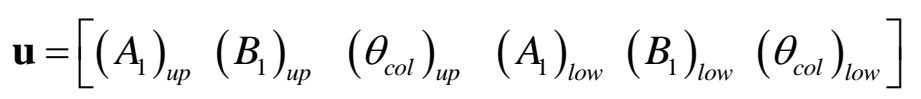

\subsection{Stability Analysis}

Stability analysis is one of the key concerns to permit expression of cause and effect in parametric form in helicopter research following a disturbance from trim condition. The nature modes of motion are indicated by the eigenvalues of the stability matrix in the Table 4.

Table 4. Eigenvalues of the Model in Hovering Condition

\begin{tabular}{c|c}
\hline eigenvalues & Nature mode \\
\hline-0.563 & roll \\
-0.4806 & pitch \\
-4368 & heave \\
$0.2125 \pm 0.3908 \mathrm{i}$ & unstable \\
$0.6308 \pm 0.4309 \mathrm{i}$ & unstable \\
0.6925 & unstable \\
\hline
\end{tabular}

As saw from the Table 4, pitch subsidence, roll subsidence and heave subsidence mode of model exhibit stability. Since two rotors flap in opposite directions with decreasing the rotor dihedral effect on roll moments. The eigenvalue of roll mode drops and gets close to the pitch one with being homologous to the situation of closely values of roll and pitch early. The dynamic model is stable in heave mode. It is not stable in the yaw mode since to speculation on the combination of the lack of 
a tail rotor. Two complex conjugate pairs in the right half-plane are unstable oscillation. These two paires are belonging the longitudinal and lateral dynamics, respectively. This is because that the coupling of the pitch and longitudinal velocity caused by the speed stability. The other due to the coupling of roll and lateral velocity. These situation also happened in conventional helicopter. Furthermore, the distribution trend of natural modes of dynamic model coincides well with the existed results[26].

In summary, the fidelity of our simulation model presents constraints on what can be achieved from the point of view of stability analysis because of ignoring rotor dynamic motion. The more accurate the model becomes, the reliable stability analysis increases. However, the configuration of coaxial rotor with the lack of a tail rotor will intuitive play a dominant role in analysis stability when comparing the conventional helicopter. Many works of coaxial rotor helicopter aerodynamic model need to be detailed and deeply implemented, such as, the validated experimental results, but beyond the scope of this article.

\section{Simulation Results}

It was implemented to investigate the dynamic response characteristic under the proposed trim method. Since the proposed trim method was different from trimming strategy that was similar adopted in the conventional helicopter, different stability and control derivatives comes into being and dynamic response most likely has changed. Consequently, a suggestive control allocation strategy of the coaxial rotor helicopter is concluded after investigating the singularity response of each input comprehensively and objectively. It also provides a foundation to control this model.

Figure 2 and 3 , pitch $\theta$ and pitch rate are clearly affected by longitudinal cyclic pitch $\left(B_{1}\right)_{\text {low }}$ and $\left(B_{1}\right)_{u p}$. Figure $2 \mathrm{a}$ and Figure $3 \mathrm{a}$ point out that longitudinal cyclic pitch of upper rotor and lower rotor effects in pitch and pitch rated in different frequency bandwidth. There is considerable coupling of the forces and moments introduced by pitch moments between pitch and pitch rate due to control pitch moments. It must be first change pitch rate then attitude. In Figure 2, it indicates that longitudinal cyclic pitch $\left(B_{1}\right)_{\text {low }}$ can be used to $\operatorname{control} \theta$. Except pitch $\theta$, upper rotor's longitudinal cyclic pitch $\left(B_{1}\right)_{u p}$ is more effective in pitch rate $q$ than other control variables in high frequency in Figure 3. Therefore, the upper rotor's longitudinal cyclic can be used to control the pitch rate.

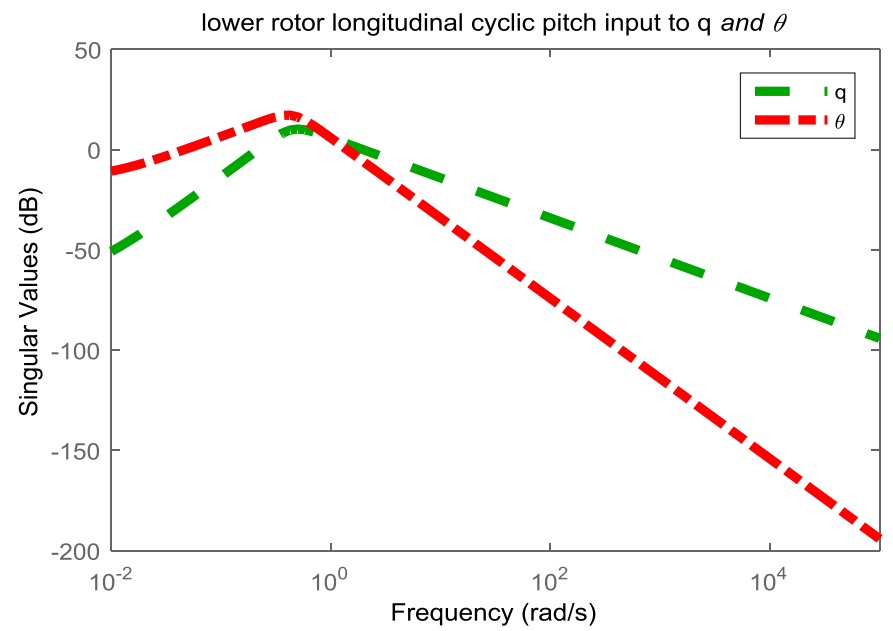

(a) 


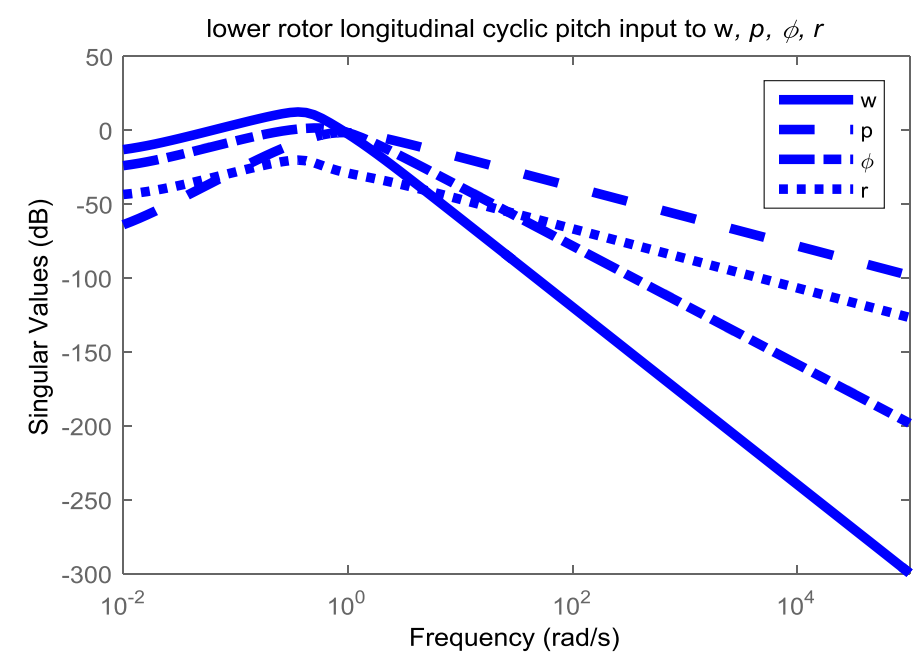

(b)

Figure 2. Singular Value Responses of Lower-rotor Longitudinal Cyclic

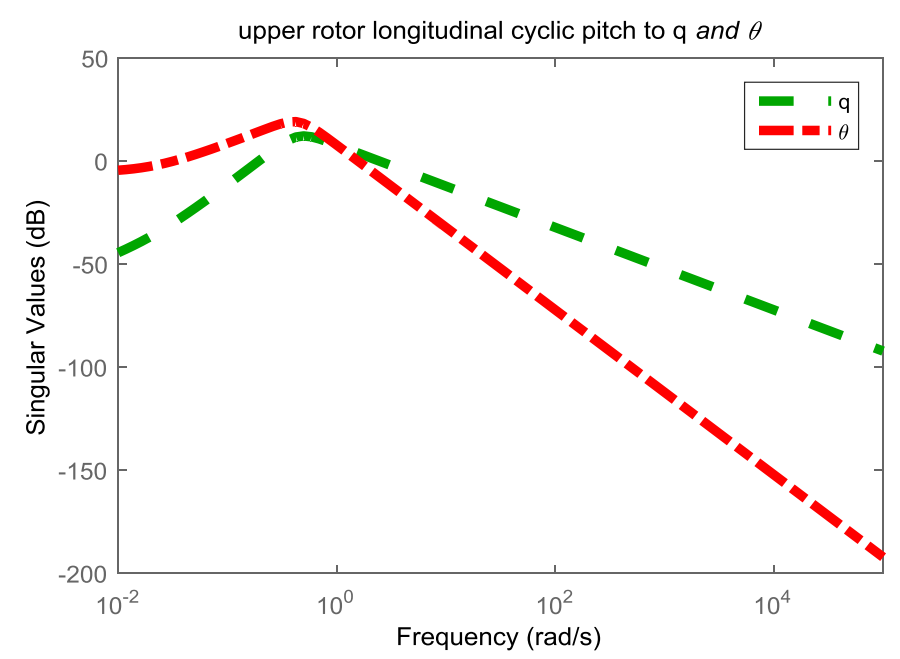

(a)

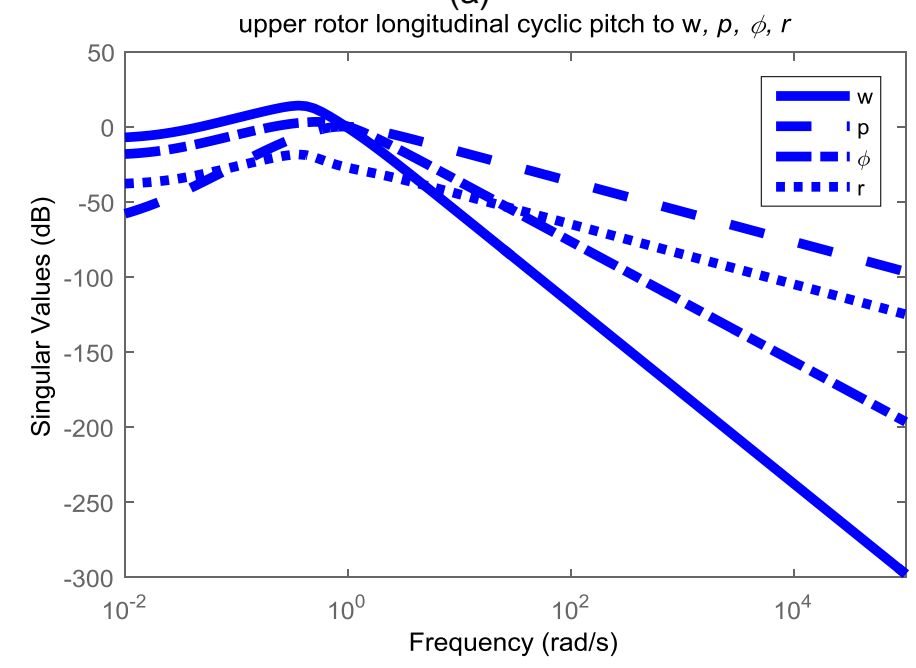

(b)

Figure 3. Singular Value Responses of Upper-rotor Longitudinal Cyclic 
In Figure 4 and Figure 5, they indicate roll $\phi$ and roll rate are clearly affected by lateral cyclic pitch $\left(A_{1}\right)_{\text {low }}$ and $\left(A_{1}\right)_{u p}$. There is also considerable coupling between the roll and roll rate. It points out that lateral cyclic pitch of upper $r$ and lower rotor effects in roll and roll rated in different frequency bandwidth in Figure 4a and Figure 5a. Therefore, it indicates that $\left(A_{1}\right)_{\text {low }}$ can be used to control roll at low frequency. Upper rotor's lateral cyclic pitch $\left(A_{1}\right)_{u p}$ is more effective in roll $\phi$ than pitch $\theta$ in Figure 4. Except roll, $\left(A_{1}\right)_{u p}$ is more effective in roll rate $p$ than other control variables as the same as $\left(A_{1}\right)_{\text {low }}$ effects pitch rate $q$ in Figure 6.

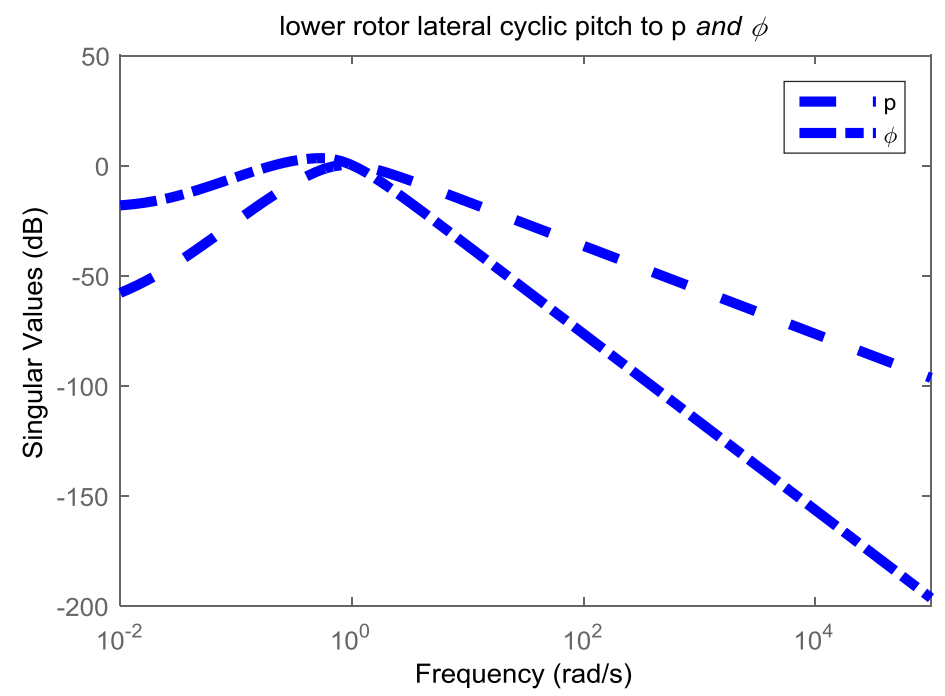

(a)

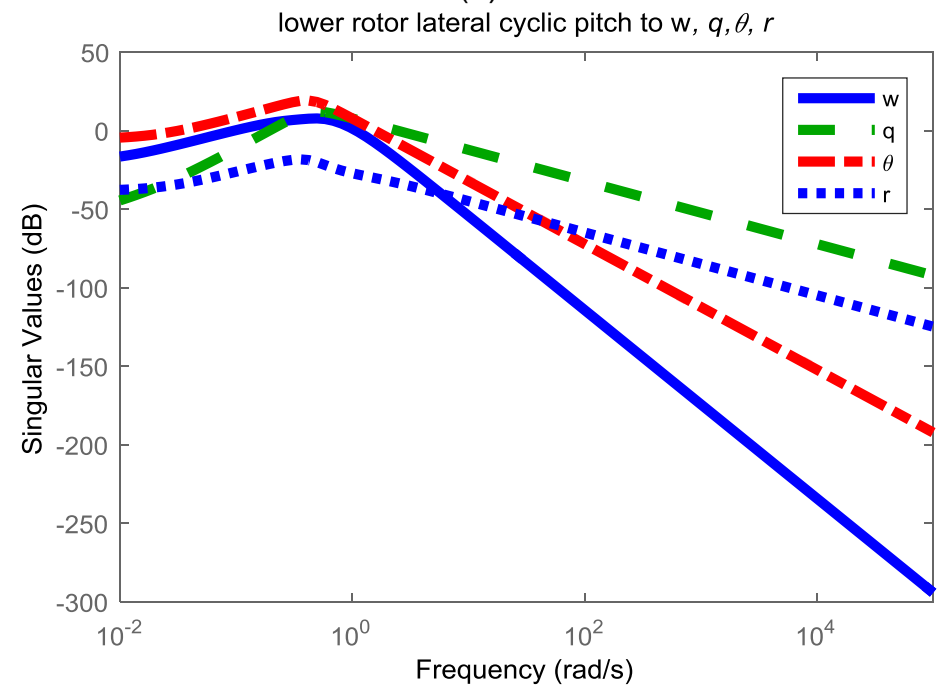

(b)

Figure 4. Singular Value Responses of Lower-rotor Lateral Cyclic

As saw from Figure 2, 3, 4 and 5, there are inherently cross-couplings between longitudinal and lateral motions due to axis symmetry of rigid-body. It also indicates that the constructed dynamic model is reasonable in depicting basic dynamic motion of helicopter. In addition, the heave and yaw state also effect the longitudinal and lateral 
control variables. Moreover, it also indicated that the upper inputs more effective on states and less complicated state coupling than the lowers in the Figure2a, 3a when comparing with Figure 4a, 5a. This difference corresponding to the trim method based on the thrust ratio.

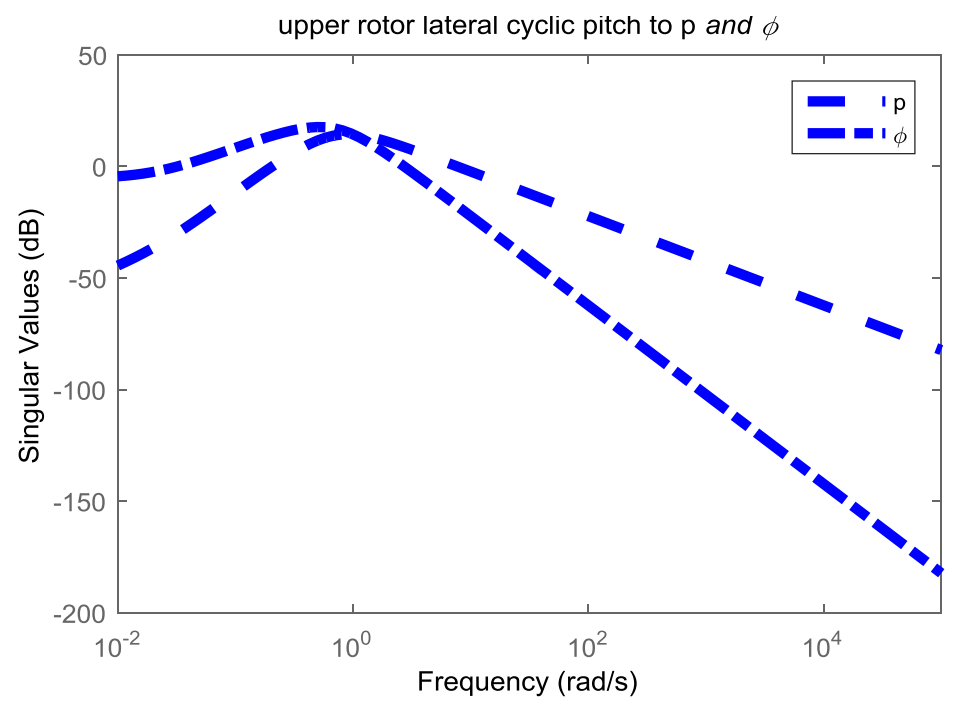

(a)

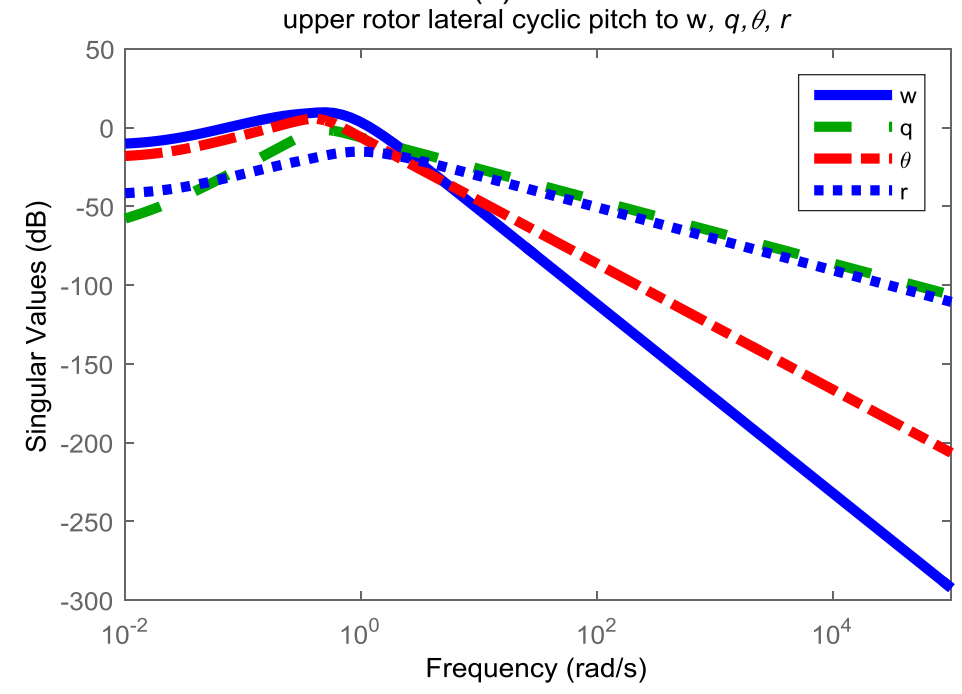

(b)

Figure 5. Singular Value Responses of Upper-rotor Lateral Cyclic

It shows that lower rotor's collective pitch significantly affects heave and yaw motion in the Figure 6. Hence, seriously coupling effects between heave and yaw motion are present. Furthermore, lower rotor's collective $\left(\theta_{c o l}\right)_{\text {low }}$ and upper rotor's collective $\left(\theta_{c o l}\right)_{u p}$ all more effective in vertical velocity $w$ than yaw rate $r$ and than the other control variables. Then, it is possible to control vertical velocity using lower rotor's collective $\left(\theta_{c o l}\right)_{\text {low }}$ or upper rotor's collective $\left(\theta_{c o l}\right)_{u p}$, the rest input can will be used to control yaw rate $r$. Seriously coupling between heave and yaw motion in the constructed dynamic model. 


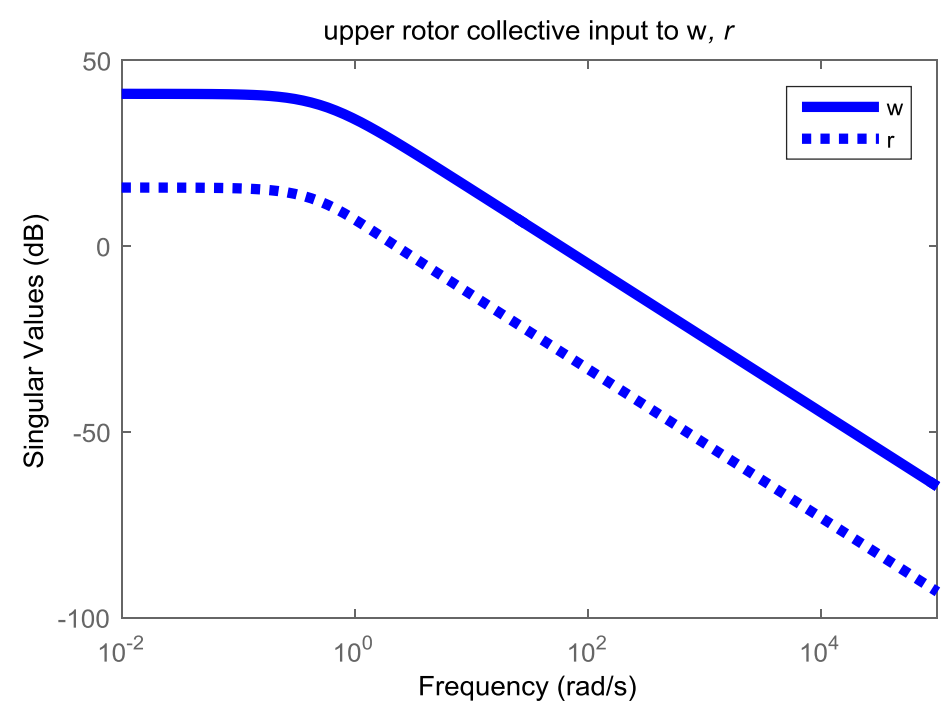

(a)

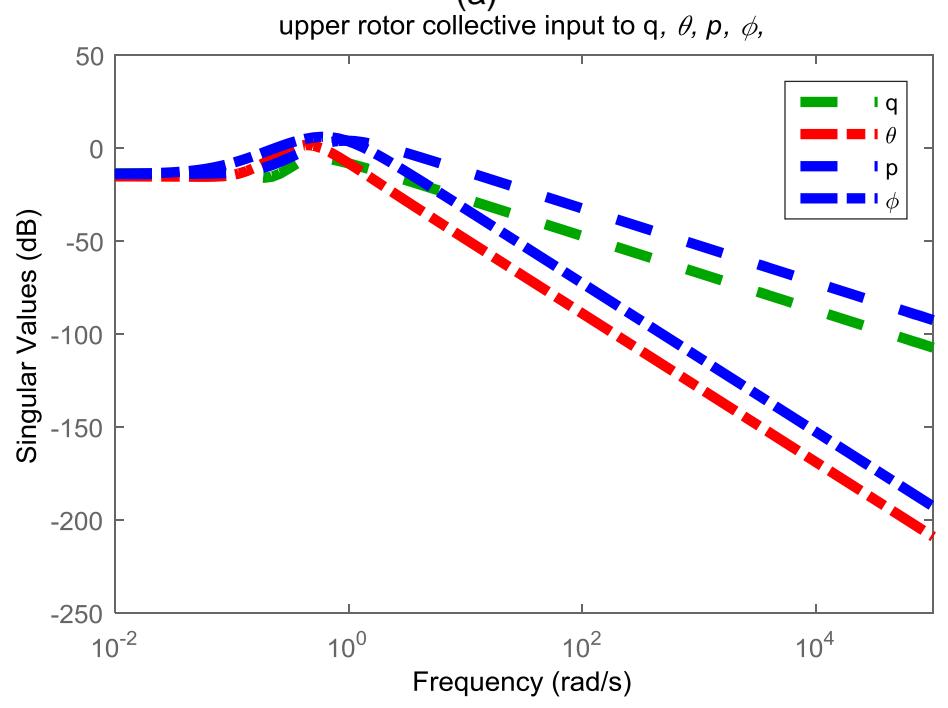

(b)

Figure 6. Singular Value Responses of Upper-rotor Collective

As saw from Figure 2 to Figure 7, the couplings between longitudinal-lateral dynamic and heave-direction dynamic still interference each other. Furthermore, Figure 2 to Figure 7 reveal that any one input effect at least two control variables. Upper rotor's collective input even has influence on all of six control variables as illustrated by Figure 3 Furthermore, plant has a high condition number at some frequencies reflected on its sensitivity to a relative input change.

In summary, the singularity responses simulations show that the model experiences complicated couplings among channels. The control scheme is concluded as diagonally dominant as possible in Table 5 . 


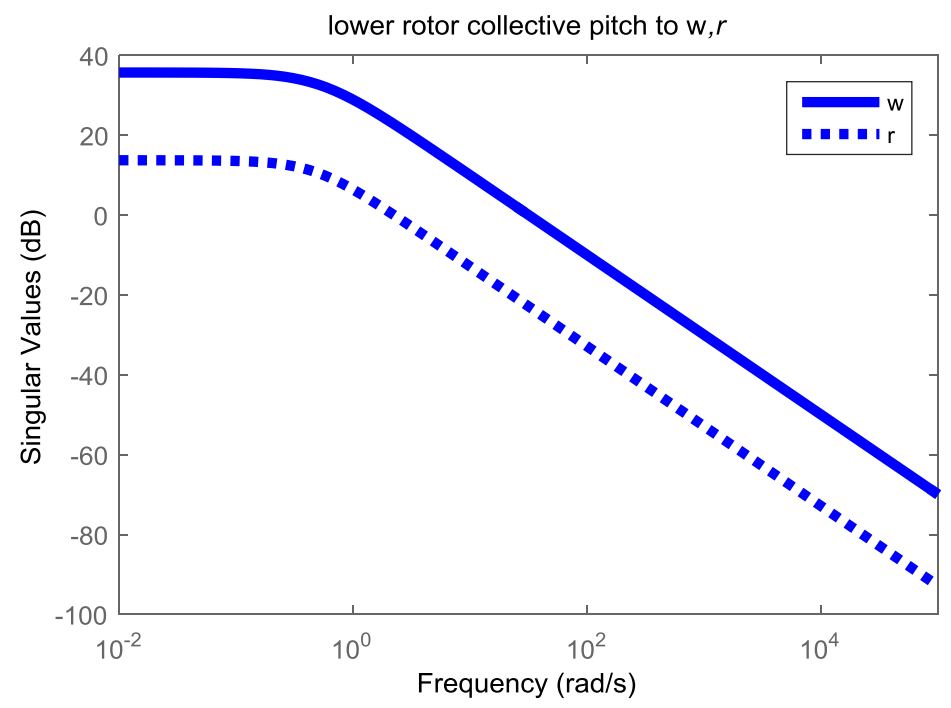

(a)

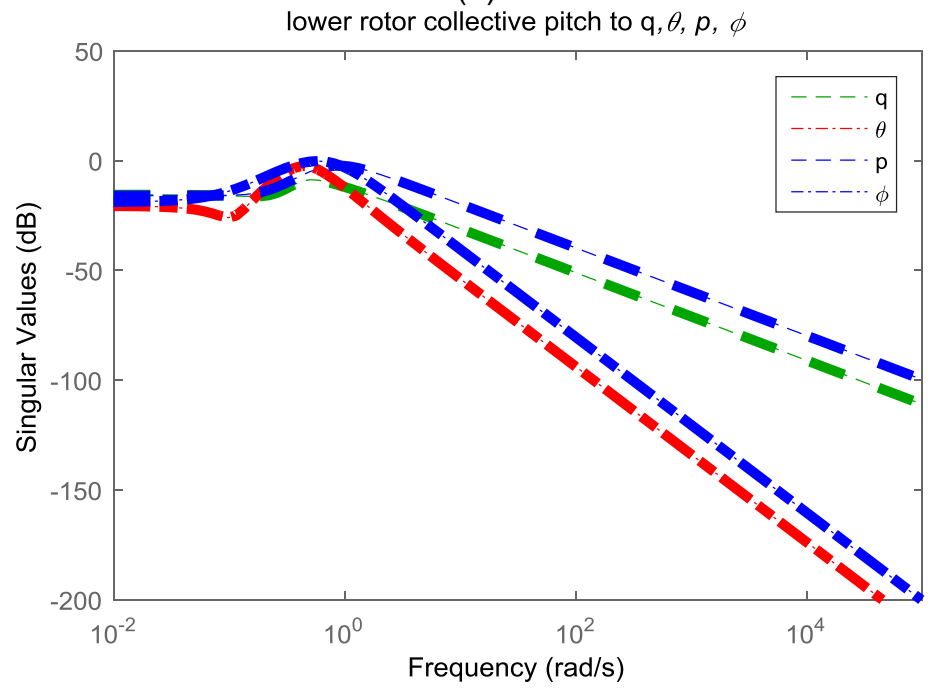

(b)

Figure 7. Singular Value Responses of Lower-rotor Collective

Table 5. Suggestive Control Allocation Strategy of the Coaxial Helicopter

\begin{tabular}{l|l|l|l|l|l|l}
\hline $\begin{array}{l}\text { Input } \\
\text { degree }\end{array}$ & $\begin{array}{l}\text { longitudinal } \\
\left(B_{1}\right)_{\text {low }}\end{array}$ & $\begin{array}{l}\text { longitudinal } \\
\left(B_{1}\right)_{u p}\end{array}$ & $\begin{array}{l}\text { Lateral } \\
\left(A_{1}\right)_{\text {low }}\end{array}$ & $\begin{array}{l}\text { Lateral } \\
\left(A_{1}\right)_{u p}\end{array}$ & $\begin{array}{l}\text { Collective } \\
\left(\theta_{\text {col }}\right)_{\text {low }}\end{array}$ & $\begin{array}{l}\text { Collective } \\
\left(\theta_{\text {col }}\right)_{u p}\end{array}$ \\
\hline $\begin{array}{l}\text { Control } \\
\text { variables }\end{array}$ & Pitch $\theta$ & Pitch rate $q$ & Roll $\phi$ & $\begin{array}{l}\text { Roll } \\
\text { rate } p\end{array}$ & Yaw rate $r$ & $\begin{array}{l}\text { Heave } \\
\text { rate } w\end{array}$ \\
\hline
\end{tabular}

\section{Conclusions}

A trimming methodology was proposed to utilize high efficiency upper rotor to change the coaxial rotor's moments based on the different rotors' thrust ratio. This methodology includes modeling method, trim strategy, figure-of-merit analysis and simulation method. The integration method was advocated to construct mathematical model. Frequency response analysis was implemented to investigate the dynamic response characteristic of the constructed model. Then, dynamic characteristic of proposed trim strategy were compared with the existed research. Effects of proposed 
trimming strategy on trim values were also discussed deeply. Frequency response simulations prove that the simulation model can bring an appropriate representation of dynamic response characteristic under trimming methodology. A suggestive control allocation strategy of the coaxial rotor helicopter is concluded. It is observed that the upper inputs more effective on states and less complicated state coupling than the lowers uppers. The possession of dominate upper rotor cyclic input with high figure of merit show a confident guide to contribute to a less complicate and lighter coaxial rotor system. In addition, It also provides more flexible input than the previous work. It also provides a foundation to control this model. This trim methodology also can be easily extend to other flight regime.

\section{Acknowledgments}

This project was funded by National High Technology Research and Development Program 863 of China (No.2013-AA063903), the Research Fund for the Doctoral Program of Higher Education of China (Grant No: 20130061120038), Young Teacher Innovation Project of Jilin University of China (Grant No:450060491425) and China Postdoctoral Science Foundation (Grant No:2014M560232).The first author are very grateful to his advisor for supporting part of this project.

\section{References}

[1] M. Syal and J. G. Leishman, "Aerodynamic Optimization Study of a Coaxial Rotor in Hovering Flight", Journal of the American Helicopter Society, vol. 57, no. 4, (2012), pp. 1-15.

[2] S. Joseph and C. Inderjit, "Performance and Loads Prediction for a High Advance Ratio Coaxial Rotor", 56th AIAA/ASCE/AHS/ASC Structures, Structural Dynamics, and Materials Conference. American Institute of Aeronautics and Astronautics, (2015).

[3] M. Ramasamy, "Hover Performance Measurements Toward Understanding Aerodynamic Interference in Coaxial, Tandem, and Tilt Rotors", Journal of the American Helicopter Society, vol. 60, no. 3, (2015), pp. 1-17.

[4] R. W. Prouty and H. C. Curtiss, "Helicopter control systems: A history", Journal of Guidance Control and Dynamics, vol. 26, no. 1, (2003), pp. 12-18.

[5] H. Gao, O. Kaynak, T. Kubota, R. Sandau and E. Tunstel, "Guest Editorial Focused Section on Aerospace Mechatronics”, Mechatronics, IEEE/ASME Transactions on, vol. 18, no. 4, (2013), pp. 12331236.

[6] P. David and B. Dinesh, "A general theory of rotorcraft trim”, 36th Structures, Structural Dynamics and Materials Conference. American Institute of Aeronautics and Astronautics, (1995).

[7] J.O'Leary, and W. Miao, "Design of Higher Harmonic Control for the ABC", the American Helicopter Society Mideast Region National Specialists' Meeting on Rotor System Design. Philadelphia, (1980), pp. 52-57.

[8] H. W. Kim, A. R. Kenyon, R. E. Brown and K. Duraisamy, "Interactional aerodynamics and acoustics of a hingeless coaxial helicopter with an auxiliary propeller in forward flight", Aeronautical Journal, vol. 113, no. 1140, (2009), pp. 65-78.

[9] C. P. Coleman, "A Survey of Theoretical and Experimental Coaxial Rotor", (1997).

[10] J. Lee, S. Chae, S. Oh and K. Yee, "Parametric Study for Hovering Performance of a Coaxial Rotor Unmanned Aerial Vehicle", Journal of Aircraft, vol. 47, no. 5, (2010), pp. 1517-1530.

[11] Y. Xiaming and Z. Jihong, "Comprehensive Modeling and Analysis of an Unmanned Coaxial Helicopter", AIAA Guidance, Navigation, and Control Conference. American Institute of Aeronautics and Astronautics, (2015).

[12] J. W. Lim, K. W. McAlister and W. Johnson, "Hover Performance Correlation for Full-Scale and Model-Scale Coaxial Rotors", Journal of the American Helicopter Society, vol. 54, no. 3, (2009), pp. 032005 .

[13] J. G. Leishman and S. Ananthan, "An Optimum Coaxial Rotor System for Axial Flight", Journal Of the American Helicopter Society, vol. 53, no. 4, (2008), pp. 366-381.

[14] Y. Cao, L. Cao and S. Wan, "Trim Calculation of the CH-53 Helicopter Using Numerical Continuation Method", Journal of Guidance, Control, and Dynamics, vol. 37, no. 4, (2014), pp. 1343-1349.

[15] F. D. Kim, R. Celi and M. B. Tischler, "Forward flight trim and frequency response validation of a helicopter simulation model", Journal of Aircraft, vol. 30, no. 6, (1993), pp. 854-863.

[16] C. R. Theodore and M. B. Tischler, "Development and Operation of an Automatic Rotor Trim Control System for the UH-60 Individual Blade Control Wind Tunnel Test", Presented at the American Helicopter Society Specialists Conference on Aeromechanics. San Francisco, CA, (2010). 
[17] F. Kendoul, "Survey of advances in guidance, navigation, and control of unmanned rotorcraft systems", Journal of Field Robotics, vol. 29, no. 2, (2012), pp. 315-378.

[18] D. J. Walker, "Multivariable control of the longitudinal and lateral dynamics of a fly-by-wire helicopter", Control Engineering Practice, vol. 11, no. 7, (2003), pp. 781-795.

[19] Z. Petrovic, S. Stupar, I Kostic and A. Simonovic, "Determination of a Light Helicopter Flight Performance at the Preliminary Design Stage", Strojniski Vestnik-Journal of Mechanical Engineering, vol. 56, no. 9, (2010), pp. 535-543.

[20] I. Postlethwaite, E. Prempain, E. Turkoglu, M. C. Turner, K. Ellis and A. W. Gubbels, "Design and flight testing of various controllers for the Bell 205 helicopter", Control Engineering Practice, vol. 13, no. 3, (2005), pp. 383-398.

[21] P. Raymon, "Helicopter Performance, Stability and Control”, Krieger Publishing Company, (2002).

[22] G. D. Padfield, "Helicopter flight dynamics the theory and application of flying qualities and simulation modelling", Blackwell Publishing, (2007).

[23] R. Edward and T. A. Egolf, "Coaxial Rotor Wake and Prop Induction Impact on a Horizontal Tail Using HPCMP CREATE TM-AV Helios", 53rd AIAA Aerospace Sciences Meeting. American Institute of Aeronautics and Astronautics, (2015).

[24] J. G. Leishman and M. Syal, "Figure of merit definition for coaxial rotors", Journal Of the American Helicopter Society, vol. 53, no. 3, (2008), pp. 290-300.

[25] V. K. Lakshminarayan and J. D. Baeder, "High-Resolution Computational Investigation of Trimmed Coaxial Rotor Aerodynamics in Hover", Journal of the American Helicopter Society, vol. 54, no. 4, (2009), p. 042008.

[26] K. Ferguson and D. Thomson, "Flight Dynamics Investigation of Compound Helicopter Configurations", Journal of Aircraft, vol. 52, no. 1, (2015), pp. 156-167.

\section{Authors}
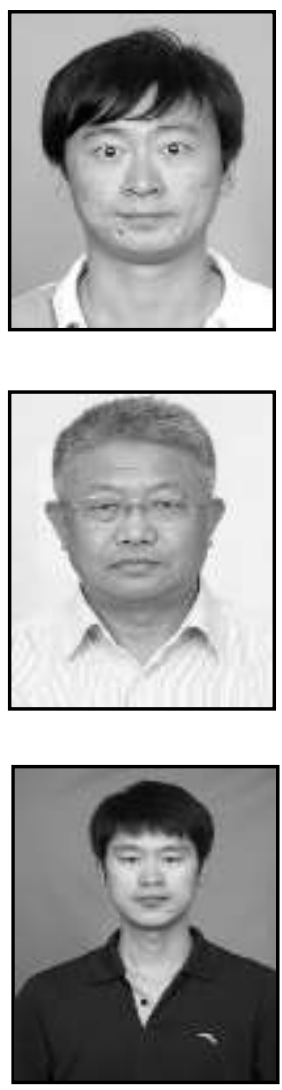

Yanli Chen, he is associate professor at School of Mechanical Science and Engineering, Jilin University, China. He received his $\mathrm{Ph} . \mathrm{D}$. in Mechanical Engineering from Jilin University in 2012. His research interests include robot, robust control, intelligent control and AUV design. 


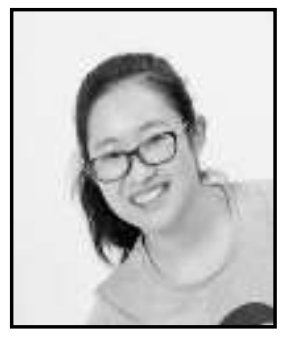

Jialin Liu, he is associate professor at School of Mechanical Science and Engineering, Jilin University, China. He received his $\mathrm{Ph} . \mathrm{D}$. in Mechanical Engineering from Jilin University in 2012. His research interests include robot, robust control, intelligent control and AUV design.



Wenjian Wu, he works at SAIC-GM-Wuling Automobile Co., Ltd, China. He received his M.S in Mechanical Engineering from Jilin University in 2015. His research interests include robot, modeling, numerical simulation and rotorcraft design. 
International Journal of Control and Automation Vol.10, No.8 (2017) 